\title{
A REMARK ON BOUNDARY RESTRICTIONS IN THE GLOBAL NEWTON METHOD*
}

\author{
Hal R. VARIAN \\ University of Michigan, Ann Arbor, MI 48109, U.S.A.
}

Received May 1976

This remark shows how Smale's method of computing fixed points can be extended to problems involving very general boundary behavior.

\section{Introduction}

Consider a smooth vector field on the unit disk defined by $\dot{x}=f(x)$, $f: D^{n} \rightarrow R^{n}$. If this vector field 'points inward' on the boundary of the disk, then it is well known that there exists an equilibrium $x^{*}$ where $f\left(x^{*}\right)=0$.

Recently, Smale (1976) and Kellog, Li, and Yorke (1976) have suggested algorithms to compute such an equilibrium. In particular, Smale has shown that if the vector field satisfies some rather strong boundary conditions, there is a differential equation whose solution tends to some equilibrium $x^{*}$ when one starts at almost any point on the boundary of $D^{n}$. The differential cquation is given implicitly by

$$
D f(x) \frac{\mathrm{d} x}{\mathrm{~d} t}=-\lambda f(x),
$$

where $\lambda$ is an arbitrary scalar function of $x$ such that $\operatorname{sign} \lambda=\operatorname{sign} \operatorname{det} D f(x)$.

Smale's boundary conditions are rather complicated to state and in any practical problem they may be difficult to verify. The purpose of this note is to show how these boundary conditions can be significantly relaxed. In particular, I show that under the condition that $f(x)$ points inward on the boundary of $D^{n}$ one can find an equilibrium by following the above differential equation through the use of an appropriate trick.

*This research was funded in part by National Science Foundation grant number SOC7609414. I wish to thank Steve Smale for some helpful conversations. 


\section{The method}

Let us suppose initially that the vector field meets the very strong condition that it points radially inward on the boundary of $D^{n}$, that is, $f(x)=-x$ on the boundary of $D^{n}$. First, I will outline a description of Smale's method in this simple case, and then show how the general case may be modified so as to fit into this framework.

Define $M$ to be the unit disk excluding the set of equilibria. Then the 'Gauss map' $g: M \rightarrow S^{n-1}$ given by $g(x)=f(x) /\|f(x)\|$ is well defined. Sard's theorem shows that for almost all $e$ in $S^{n-1}, g^{-1}(e)$ is a one-dimensional manifold; that is, a finite union of circles and line segments with the boundaries of the segments coinciding with the boundary of $M$.

Now, since $f(x)=-x$ on the boundary of $D^{n}$, there is one and only one point $x_{e}$ on this boundary in $g^{-1}(e)$. But the other end of the line segment starting at $x_{e}$ must lead to a point on the boundary of $M$. Therefore it must lead to an equilibrium.

Let us parameterize this path by $x(t)$; by the definition of $g, x(t)$ must satisfy the identity

$$
f(x(t)) \equiv e\|f(x(t))\|
$$

Differentiating this with respect to $t$ gives

$$
D f(x(t)) \frac{\mathrm{d} x}{\mathrm{~d} t}=e a(t)=\frac{f(x(t)) a(t)}{\|f(x(t))\|}=f(x(t)) \lambda(t),
$$

where $a(t)$ and $\lambda(t)$ are some scalar constants; since they only indicate the speed at which we move along the path, their magnitude is unimportant. However, the sign of $\lambda(t)$ is important. An orientation argument shows that the appropriate sign is that of det $-D f(x)$. Thus if we start at almost any point on the boundary of $D^{n}$ and follow the differential equation given above, we will be led to an equilibrium.

The derivation is exactly the derivation used by Smale applied in the special case where $f(x)=-x$ on the boundary of $D^{n}$. Smale's boundary assumption was actually somewhat weaker than this; it essentially implied that the Gauss map defined above is one-to-one on the boundary of the disk. This means that there are no two points $x$ and $y$ on the boundary of the disk where $f(x)$ and $f(y)$ point in the same direction. When stated in this way Smale's boundary assumption seems very restrictive.

It turns out that this boundary assumption can be relaxed significantly. In fact, one only needs to assume that the vector field defined by $f(x)$ never points radially outwards on the boundary of the disk: 
Boundary Condition. At all $x$ on the boundary of $D^{n}$, there is no positive scalar $r$ such that $f(x)=r x$.

It is clear that this condition is compatible with the requirement that $f(x)$ points inward on the boundary of $D^{n}$. We now reduce this general boundary condition to the previous case.

Let $D_{2}^{n}$ be a disk in $R^{n}$ of radius 2 . Let $s=\|x\|-1$ and define the following vector field on $D_{2}^{n}$ :

$$
\begin{aligned}
h(x) & =-\frac{s x}{\|x\|}+(1-s) f\left(\frac{x}{\|x\|}\right), & & 1<\|x\| \leqq 2, \\
& =f(x), & & 0 \leqq\|x\| \leqq 1 .
\end{aligned}
$$

This field coincides with the original field on the unit disk and is a continuous extension on $D_{2}^{n} \backslash D^{n}$. In fact, a vector in this portion of $D_{2}^{n}$ is simply a linear combination of the vector $x /\|x\|$ and $f(x /\|x\|)$ with the weights being given by the radial distance. The important thing to note is that this extension introduces no new cquilibria. For suppose $x^{*}$ in $D_{2}^{n} \mid D^{n}$ were such that $h\left(x^{*}\right)=0$; then

$$
\begin{aligned}
& \frac{-s x^{*}}{\left\|x^{*}\right\|}+(1-s) f\left(\frac{x^{*}}{\left\|x^{*}\right\|}\right)=0, \quad 0<s<1, \\
& f\left(\frac{x^{*}}{\left\|x^{*}\right\|}\right)=\frac{s}{(1-s)} \frac{x^{*}}{\left\|x^{*}\right\|},
\end{aligned}
$$

but this contradicts the assumption that no vector on the boundary of $D^{n}$ points radially outward. It is therefore clear that Smale's method can be applied directly to the above system.

\section{Some remarks on the method}

(1) There is a slight problem which was pointed out to me by James Mirlees. The extension defined above is not differentiable in a radial direction at the boundary of $D^{n}$. However, Smale's proof can easily be modified to take account of such a well behaved non-differentiability. The essential step is to choose $e$ to be a regular value of $g$ restricted to $D^{n}, g$ restricted to $D_{2}^{n} \backslash D^{n}$, and $g$ restricted to the boundary of $D^{n}$. This ensures that $g^{-1}(e)$ will intersect the boundary of $D^{n}$ transversely and will therefore be a 1-manifold with a finite number of kinks. Hence Smale's differential equation will still be well defined as long as one chooses the correct Jacobian matrix when crossing the boundary of $D^{n}$. 
(2) The boundary condition described above is very natural from an economic point of view. Nishimura (1976) has shown that if one has the condition of free disposal and the assumption of no boundary equilibria, it follows that the vector of excess demand can never point radially outward on the boundary of the price simplex.

(3) Some geometrical insight into the method can be gained by examining fig. 1. By construction, the vector $f(x)$ points in the direction $e$ at each $x$ along the path $g^{-1}(e)$. If thcre are two points on the boundary of $D^{n}$ where $f(x)$ points in the direction $e$ they may be connected by a component of $g^{-1}(e)$. However, degree considerations show that there must be some component of $g^{1}(e)$ that starts at the boundary of $D^{n}$ and leads to an equilibrium. The extension suggested here gives a way of finding such a path.

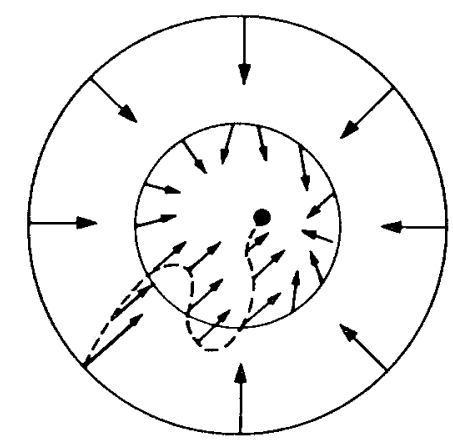

Fig. 1. The vectors along the path $g^{-1}(e)$ are all parallel to each other.

\section{References}

Smale, S., 1976, Convergent processes of price adjustment and Global Newton methods, Journal of Mathematical Economics 3, 107-120.

Kellog, B., T.Y. Li and J. Yorke, 1976, A method of continuation for calculating a Brouwer fixed point, in: S. Karamdiar, ed., Computing fixed points with applications (Academic Press, New York) 3, 107-120.

Nishimura, K., 1976, A further remark on the number of equilibria of an economy, University of Rochester Discussion Paper (Rochester, NY); International Economic Review, to appear. 